\title{
CHARGE DISTRIBUTION ON THIN SEMICONDUCTING SILICON NANOWIRES
}

\author{
Hui Chen and Subrata Mukherjee \\ Department of Theoretical and Applied Mechanics \\ Kimball Hall, Cornell University \\ Ithaca, NY 14853 \\ Narayan Aluru \\ Department of Mechanical Science and Engineering \\ University of Illinois at Urbana-Champaign \\ Urbana, Illinois 61801
}

February 6, 2008

\section{CMAME-D-07-00585 Accepted for publication in CMAME}

keywords: charge distribution, semiconducting silicon nanowires, boundary element method, finite element method

\begin{abstract}
The subject of this paper is the calculation of charge distribution on and inside thin semiconducting silicon nanowires in electrostatic problems, by a coupled Finite and Boundary Element Method (FEM/BEM). A hybrid semiclassical (Laplace/Poisson) model is employed and a line model (with finite thickness) for a silicon nanowire of circular cross-section is proposed here. This model overcomes the problem of dealing with nearly singular matrices that occur when the standard BEM is applied to very thin features (objects or gaps). This new approach is also very efficient. Numerical results are presented for selected examples.
\end{abstract}




\section{Introduction}

Microelectromechanical systems (MEMS) have demonstrated important applications in a wide variety of industries including mechanical and aerospace, medicine, communications, information technology etc. Nanoelectromechanical systems (NEMS) are "smaller" MEMS in the sense that they have submicron critical dimensions. Owing primarily to their small size, NEMS can offer very high sensitivities (e.g. force sensitivities at the attonewton level, mass sensitivities at a single molecule or even a single atom level, and charge sensitivities at the level of the charge on a single electron). In addition, they offer mechanical quality factors in the tens of thousands and fundamental frequencies in the microwave range ([1] - [5]). Mechanical resonance and losses in nanometer scale silicon wires have been measured [6]. Fabrication of silicon nanotweezers [7] and nanoresonators [8] has been demonstrated recently.

Numerical simulation of electrically actuated MEMS devices have been carried out for nearly fifteen years by using the Boundary Element Method (BEM - see, e.g. [9, 10, 11, 12, 13]) to model the exterior electric field and the Finite Element Method (FEM - see, e.g. [14, 15, 16]) to model deformation of the structure. The commercial software package MEMCAD [17], for example, uses the commercial FEM software package ABAQUS for mechanical analysis, together with a BEM code FastCap [18] for the electric field analysis. Other examples of such work are [19, 20, 21, 22]; as well as $[17,23,24]$ for dynamic analysis of MEMS. A recently published paper [25] addresses the problem of charge distribution on multiwalled nanotubes, with a classical electrostatics model, by employing a full three-dimensional (3-D) BEM approach.

MEMS have been analyzed using classical continuum models for the mechanical (elastostatic or elastodynamic) and electrical (electrostatic) energy domains. These physical models for MEMS, however, may not be directly applicable to NEMS because of their small sizes. Mechanical and electrical behavior, as well as the coupling between domains, could be different for nanoscale structures, thus requiring different physical models.

Electrostatic analysis of MEMS assumes that the microstructures can be treated as conductors. In such cases, the potential in a conductor is uniform, all the charges distribute on the surfaces of the conductors and the coupling between the electrical and mechanical domains takes place through the electrostatic forces which act only on the surfaces of the conductors. The conductor assumption may not hold for some NEM devices. Silicon microstructures, for example, are more appropriately treated as semiconductors. In such cases, the potential distribution in a semiconductor is no longer uniform and the charges are distributed not only on the surfaces but also in the bulk of semiconductors. When the charges are distributed throughout a semiconductor, the electrical and mechanical domains are coupled not only through the surface but also through the volume of a semiconductor. Aluru and his co-workers, in recent papers [26, 27], have employed a classical electrostatic model for conductors, and, depending upon their characteristic length, two different electrostatic models : semiclassical (Laplace/Poisson) and quantum mechanical (Laplace/Poisson/Schrödinger) for semiconductors. Numerical results for two-dimensional (2-D) problems are presented in these papers.

A recent paper [28] describes the study of the charge distribution on thin conducting carbon nanotubes (CNTs). This requires BEM analysis of the electric field exterior to these thin conducting objects. In the context of MEMS with very thin beams or plates, a convenient way to model such a problem is to assume plates with vanishing thickness and solve for the sum of the charges on the upper and lower surfaces of each plate [29]. The standard Boundary Integral Equation (BIE) with a weakly singular kernel is used in [29] and this approach works well for determining, for example, the capacitance of a parallel plate capacitor. For MEMS calculations, however, one must obtain the charge densities separately on the upper and lower surfaces of a plate since the traction at a surface 
point on a plate depends on the square of the charge density at that point. The gradient BIE is employed in [30] to obtain these charge densities separately. The formulation given in [30] is a BEM scheme that is particularly well-suited for MEMS analysis of very thin plates - for $h / L \leq .001$ - in terms of the length $L$ (of a side of a square plate) and its thickness $h$. A similar approach has also been developed for MEMS with very thin beams [31]. Similar work has also been reported recently by Chuyan et al. [32] in the context of determining fringing fields and levitating forces for 2-D beam shaped conductors in MEMS combdrives. A fully coupled BEM/FEM MEMS calculation with very thin plates has been completed [33]. See, also, [34] for an application of the thin plate idea for modeling damping forces on MEMS with thin plates.

The primary contribution of [28], therefore, is the development of a line model of a nanotube in a 3-D region (called a reduced 3-D model). This model overcomes the problem of dealing with nearly singular matrices that occur when the standard BEM is applied to very thin gaps. It also greatly simplifies the BEM calculations and is computationally very efficient. Finally, the actual charge distribution on the entire surface of a CNT can be recovered at a post-processing step!

Some issues related to the modelling of a silicon nanowire need to be clarified here. Although silicon nanowires do not have circular cross-sections, this approximation for electrostatic analysis is considered to be reasonable for the long aspect ratio nanowires that are considered in this paper. Also, size dependent mechanical properties can be incorporated into the existing framework. One way to do this is to perform molecular dynamics simulations, extract material properties, and use them in the model presented in this paper. These effects will be considered in future work.

The present paper is concerned with a geometrical situation that is similar to that considered in [28]. This time, however, the nanoscale object is a semiconducting silicon nanowire rather than a conducting carbon nanotube. A semiclassical (Laplace/Poisson) model is employed here in which the potential in the (long thin circular cross-section) semiconductor is governed by a nonlinear Poisson equation while the region exterior to the semiconductor (which extends to infinity) is governed by Laplace's equation. The FEM is used to model the potential and charge distribution on and inside the semiconducting nanowire, while the BEM is used to model the region exterior to the nanowire. The coupled FEM/BEM equations are then solved in a self-consistent manner. The large aspect ratio of the nanowire is taken advantage of to greatly simplify the analysis of this coupled, nonlinear 3-D problem; while, at the same time, (as mentioned earlier in this section) the pitfalls associated with nearly singular matrices, that can arise as a consequence of the presence of long thin structures, are carefully avoided. The result is a reduction of the full 3-D problem to a 2-D (axisymmetric) FEM problem in the nanowire (only one finite element is employed in the radial direction); and, in view of the dimensionality reduction property of the BEM for linear problems, a 2-D problem in the infinite region exterior to the nanowire.

The present paper is organized as follows. The governing equations and boundary conditions for this problem are presented first. The FEM (Galerkin) formulation for the potential and charge distribution inside the nanowire follows. BIEs are next presented for an infinite region containing one thin semiconducting nanowire and an (infinite) ground plane. The ground is modeled indirectly by adding a suitable image nanotube to the computational domain. This image tube is a nonphysical mathematical artifact that is used to guarantee zero potential on the ground plane without directly modeling it (see Section 2). Next, several sections deal with evaluation of various integrals - singular and otherwise. Some of these evaluations are rather subtle and of critical importance for obtaining the numerical results presented in this paper. The next Section describes coupling of the BEM and FEM equations. A Numerical Results Section and a Concluding Remarks Section, together with several Appendices, complete the paper. 


\section{Governing Equations}

Charge distribution on the surface of a thin conducting Single Walled Carbon Nanotube (SWNT) has been analyzed by a specialized boundary element method in [28]. Carbon nanotubes, however, are often semiconducting. Modeling of the charge distribution inside and on a semiconducting silicon nanowire is carried out in this work.

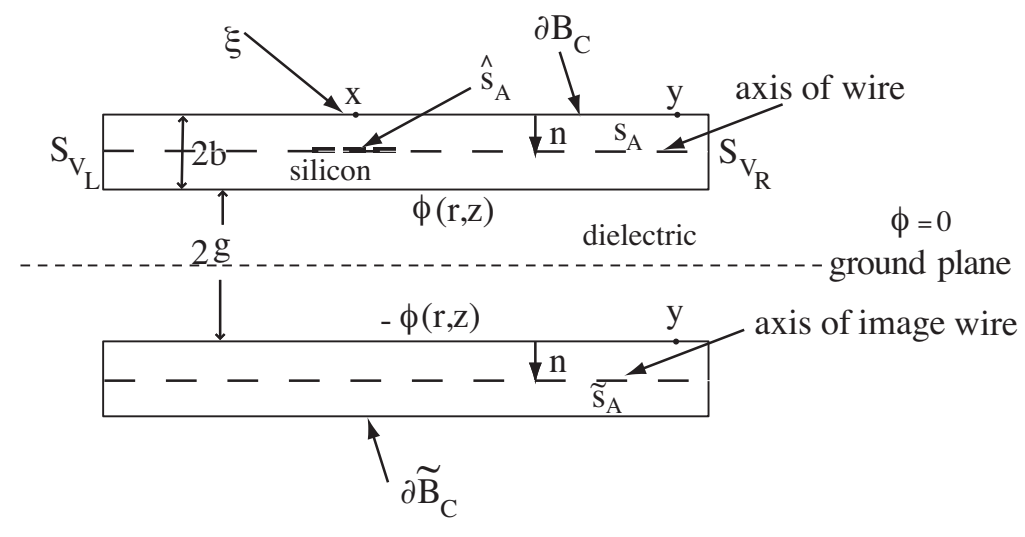

Figure 1: Silicon wire with image

The physical problem under consideration is a semi-conducting silicon nanowire with a ground plane, at zero potential, at a distance $g$ from the bottom of the wire. Fig. 1 shows a nanowire with an image in order to model the ground plane. It is important to emphasize that while the governing equation in the dielectric region outside the nanowire is linear and homogeneous (Laplace's equation), that governing the potential inside the nanowire is both nonlinear and nonhomogeneous. Therefore, linear superposition, in general, is no longer valid in this problem. The image nanowire in Fig. 1 is a nonphysical mathematical artifact which is used to guarantee zero potential on the ground plane without directly modeling it. Alternatively, the ground plane can be directly modeled with any desired potential prescribed on it. Chen and Mukherjee [35] discusses direct modeling of the ground plane for the case of conducting carbon nanotubes.

The potential inside and on a semiconducting nanowire is no longer constant, but is governed by the Poisson equation [27]. The governing equations for this problem are:

\section{Silicon.}

$$
\begin{gathered}
\epsilon_{s} \nabla^{2} \phi^{(s)}=\rho^{(s)}=-e\left[p\left(\phi^{(s)}\right)-n\left(\phi^{(s)}\right)+N_{D}^{+}\left(\phi^{(s)}\right)-N_{A}^{-}\left(\phi^{(s)}\right)\right] \\
n(\phi)=n_{0} \exp \left[\frac{e \phi}{k_{B} T}\right] \\
p(\phi)=n_{0} \exp \left[-\frac{e \phi}{k_{B} T}\right] \\
N_{D}^{+}(\phi)=N_{D}, \quad N_{A}^{-}(\phi)=N_{A}
\end{gathered}
$$




$$
n_{0}=\frac{N_{D}+N_{A}}{2}+\sqrt{\left(\frac{N_{D}+N_{A}}{2}\right)^{2}+n_{i}^{2}}
$$

\section{Dielectric}

$$
\nabla^{2} \phi^{(d)}=0
$$

\section{Boundary conditions}

$$
\begin{gathered}
\phi^{(s)}=\phi^{(d)}, \quad \text { on } \partial B_{C} \cup S_{V L} \cup S_{V R} \\
\epsilon_{s}\left(\frac{\partial \phi}{\partial r}\right)^{(s)}=\epsilon_{d}\left(\frac{\partial \phi}{\partial r}\right)^{(d)}, \quad \text { on } \partial B_{C} ; \quad \phi^{(s)}=\phi_{0} \quad \text { on } \quad S_{V L} \cup S_{V R}
\end{gathered}
$$

It is noted that the transition boundary conditions on $\partial \phi / \partial n$ in (8) at a boundary point must only be used when both $\phi$ and $\partial \phi / \partial n$ are unknown at that point.

The various quantities in (1) - (8) are as follows :

$\phi^{(s)}$ : potential in silicon, $\phi^{(d)}$ : potential in dielectric, $\rho^{(s)}$ : charge density in silicon, $\epsilon_{d}$ : dielectric constant of the dielectric medium, $\epsilon_{s}$ : dielectric constant of silicon, $n$ : electron density, $p:$ hole density, $N_{D}^{+}$: ionized donor concentration, $N_{A}^{-}$: ionized acceptor concentration, $n_{0}$ : bulk electron concentration, $n_{i}$ : intrinsic electron concentration, $e$ : charge on an electron, $k_{B}$ : Boltzmann constant, $T$ : temperature in degrees Kelvin. Also, $\partial B_{C}$ is the curved surface of the nanowire and $S_{V L}$ and $S_{V R}$ are its left and right end planes, respectively.

Some details follow. First:

$$
n=N_{C} \exp \left[\frac{E_{F}-E_{C}}{k_{B} T}\right]
$$

where $N_{C}$ is the effective density of state of the conduction band, $E_{F}$ is the Fermi-level energy and $E_{C}$ is the conduction band energy.

One has:

$$
\begin{gathered}
N_{C}=n_{i} \exp \left[\frac{\hat{E}_{C}-E_{i}}{k_{B} T}\right] \\
E_{C}=\hat{E}_{C}-e \phi^{(s)}
\end{gathered}
$$

where $\hat{E}_{C}$ is the energy of the bulk conduction band and $E_{i}$ is the intrinsic Fermi level energy.

Also:

$$
n_{0}=n_{i} \exp \left[\frac{E_{F}-E_{i}}{k_{B} T}\right]
$$

Combining (9 - 12) yields (2).

\section{Finite Element Analysis}

The potential and charge distributions inside the semiconducting nanowire are obtained by applying the finite element method. The potential inside a nanowire is assumed to be axisymmetric, i.e.

$$
\bar{\phi}^{(s)}(r, z)=\phi^{(s)}(r, \theta, z)
$$


This assumption is approximately valid as long as the nanowire is very thin and the ground plane is sufficiently far from it. It is needed in order to derive the simplified field equations that follow. A full 3-D analysis is generally required if the potential is not axisymmetric.

\subsection{Interpolation Functions and Galerkin Formulations}

The superscript $(s)$ is suppressed in this section in the interest of notational simplicity.

\subsubsection{Option one : Line model of silicon nanowire}

It is further assumed, for this section only, that the potential is a function of only the axial coordinate $z$, i.e.:

$$
\bar{\phi}(r, z)=\tilde{\phi}(z)
$$

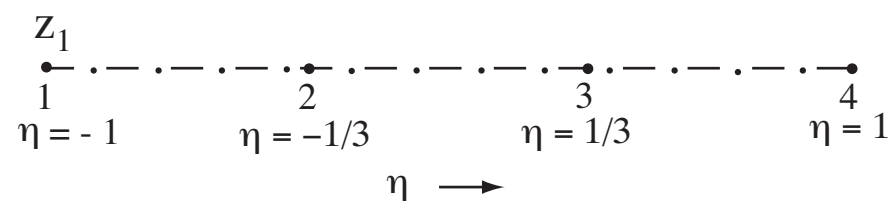

Figure 2: Finite element one. Cubic interpolations are used along the axial direction

A typical finite element for the determination of $\tilde{\phi}(z)$ is shown in Fig. 2. Piecewise cubic interpolation is used along the axis. The interpolation within a finite element, in terms of the dimensionless variable:

$$
\eta=\frac{2\left(z-z_{1}\right)}{\ell}-1
$$

has the form:

$$
\tilde{\phi}(\eta)=\sum_{k=1}^{4} N_{k}(\eta) \tilde{\phi}_{k}
$$

in terms of its nodal values $\tilde{\phi}_{k}$, and the functions $N_{k}(\eta), k=1,2,3,4$. These functions are:

$$
\begin{aligned}
& N_{1}(\eta)=-\frac{(3 \eta+1)(3 \eta-1)(\eta-1)}{16}, \quad N_{2}(\eta)=\frac{9(\eta+1)(\eta-1)(3 \eta-1)}{16} \\
& N_{3}(\eta)=-\frac{9(\eta+1)(\eta-1)(3 \eta+1)}{16}, \quad N_{4}(\eta)=\frac{(3 \eta+1)(3 \eta-1)(\eta+1)}{16}
\end{aligned}
$$

In the above, $\ell$ is the element length.

\subsubsection{Galerkin formulation for option one}

A Galerkin formulation proceeds as follows:

$$
\int_{0}^{\ell}\left[\epsilon_{s} \frac{\partial^{2} \tilde{\phi}}{\partial z^{2}}-f(\tilde{\phi})\right] N_{k}(z) d z=0
$$

In the above, $f\left(\phi^{(s)}\right)$ is the right hand side of $(1)$. 


\subsubsection{Option two : Quadratic radial variation within silicon nanowire}

A typical finite element, for the determination of $\bar{\phi}(r, z)$ is shown in Fig. 3. Piecewise cubic polynomial interpolation is used along the axis while, in view of the large aspect ratio of a typical nanowire, a single quadratic interpolation function is employed in the radial direction. This finite element is rectangular (axisymmetric with a rectangular cross-section), with length $\ell$, and extends from $r=0$ to $r=b$ in the transverse direction.

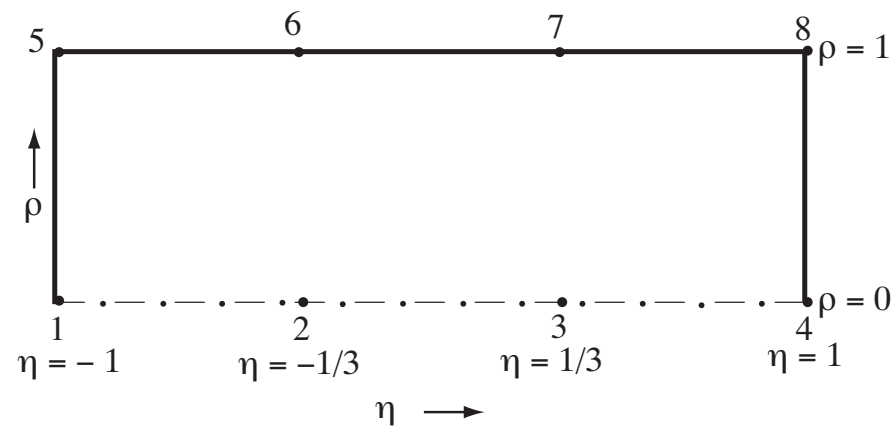

Figure 3: Finite element two. Cubic interpolations are used along the axial and a single quadratic interpolation is used in the radial direction

The interpolation within a finite element, in terms of the dimensionless variables:

$$
\rho=\frac{r}{b}, \quad \eta=\frac{2\left(z-z_{1}\right)}{\ell}-1
$$

has the form:

$$
\bar{\phi}(\rho, \eta)=\left(1-\rho^{2}\right) \sum_{k=1}^{4} N_{k}(\eta) \bar{\phi}_{k}+\rho^{2} \sum_{k=5}^{8} N_{k-4}(\eta) \bar{\phi}_{k}
$$

in terms of its nodal values $\bar{\phi}_{k}$, and the functions $N_{k}(\eta), k=1,2,3,4$. These functions are defined in (17). The special quadratic interpolation in the radial direction (20) is adopted since the nanowire is very thin compared to its length and to ensure that $\frac{\partial \phi}{\partial r}(r, z)=0$ for points on its axis (symmetry line).

\subsubsection{Galerkin formulation for option two}

A Galerkin formulation proceeds as follows:

$$
\int_{0}^{\ell} \int_{0}^{b}\left[\epsilon_{s}\left[\frac{\partial^{2} \bar{\phi}}{\partial r^{2}}+\frac{1}{r} \frac{\partial \bar{\phi}}{\partial r}+\frac{\partial^{2} \bar{\phi}}{\partial z^{2}}\right]-f(\bar{\phi})\right] M_{k}(r, z) d r d z=0
$$

In the above, $f\left(\phi^{(s)}\right)$ is the right hand side of $(1)$, and:

$$
M_{k}(\rho, \eta)=\left(1-\rho^{2}\right) N_{k}(\eta), \quad M_{k+4}(\rho, \eta)=\rho^{2} N_{k}(\eta) ; \quad k=1,2,3,4
$$




\section{Boundary Element Analysis}

This section presents BIEs for a single thin semiconducting silicon nanowire with an infinite ground plane. The ground is modelled by adding on a suitable image nanowire in the computational domain (see Fig. 1 and [28]).

Please note that the BEM needs to be collocated only on the outside nodes (i.e. on $\partial B_{C} \cup S_{V L} \cup$ $S_{V R}$ in Fig. 1). This would be nodes $1,5,6,7,8,4$ in Fig. 3. Also, by virtue of (20), axial nodes $1,2,3,4$ in Fig. 3 have $\frac{\partial \phi}{\partial r}=0$.

\subsection{Regular BIE - Source Point Approaching the Nanowire Surface $\partial B$}

For a source point $\boldsymbol{\xi} \in B \rightarrow \mathbf{x} \in \partial B=\partial B_{C} \cup S_{V L} \cup S_{V R}$ (see Fig. 1), one has:

$$
\begin{aligned}
\phi^{(d)}(\mathbf{x}) & =\int_{\partial B_{C}} \frac{\sigma^{(d)}(\mathbf{y})}{4 \pi \epsilon_{d} r(\mathbf{x}, \mathbf{y})} d S(\mathbf{y})+\int_{\partial \tilde{B}_{C}} \frac{\sigma^{(d)}(\mathbf{y})}{4 \pi \epsilon_{d} r(\mathbf{x}, \mathbf{y})} d S(\mathbf{y}) \\
& +\int_{\partial B_{C}} \frac{\mathbf{r}(\mathbf{x}, \mathbf{y}) \cdot \mathbf{n}(\mathbf{y})}{4 \pi r^{3}(\mathbf{x}, \mathbf{y})} \phi^{(d)}(\mathbf{y}) d S(\mathbf{y})+\int_{S_{V L} \cup S_{V R}} \frac{\mathbf{r}(\mathbf{x}, \mathbf{y}) \cdot \mathbf{n}(\mathbf{y})}{4 \pi r^{3}(\mathbf{x}, \mathbf{y})} \phi^{(d)}(\mathbf{y}) d S(\mathbf{y}) \\
& +\int_{\partial \tilde{B}_{C}} \frac{\mathbf{r}(\mathbf{x}, \mathbf{y}) \cdot \mathbf{n}(\mathbf{y})}{4 \pi r^{3}(\mathbf{x}, \mathbf{y})} \phi^{(d)}(\mathbf{y}) d S(\mathbf{y})+C, \quad \mathbf{x} \in \partial B
\end{aligned}
$$

In the above, $\partial B_{C}$ and $\partial \tilde{B}_{C}$ are the curved surfaces of the nanowire and of its image, respectively, and $S_{V L}$ and $S_{V R}$ are the left and right flat surfaces of the nanowire. Also, $\phi^{(d)}$ is the potential and $\sigma^{(d)}$ is the charge density per unit surface area, on the outer surface of the nanowire. The (axisymmetric) potential distribution on the nanowire is $\phi^{(d)}(r, z)$ and that on its image is $\phi^{(d)}(r, z)$. Under these conditions, $C=\phi_{\infty}=0[28]$.

It is noted that if $\mathbf{x} \in \partial B_{C}$, then the third integral in (23) must be interpreted as a finite part (FP) in the sense of Mukherjee [36] (denoted as f). Similarly, the fourth integral in (23) is an FP integral when $\mathrm{x} \in S_{V L} \cup S_{V R}$.

Define:

$$
q^{(d)}(r, z)=2 \pi b \sigma^{(d)}(r, z)
$$

where $b$ is the radius of the nanowire (see Fig. 1) and $q^{(d)}(r, z)$ is the charge density, per unit length, in the nanowire. Also, let:

$$
\bar{\phi}^{(d)}(r, z)=\phi^{(d)}(r, \theta, z), \quad \tilde{\phi}^{(d)}(z)=\bar{\phi}^{(d)}(b, z)
$$

Each term in (23) is now simplified for the special case $\ell>>b$, where $\ell$ is the length of the nanowire (Fig. 4). In view of the fact that $b$ is small, quantities $(O)\left(b^{n}\right), n \geq 2$, are neglected in the rest of this paper. It is important to realize, especially for the evaluation of integrals involving the potential in $(23)$, that $\mathbf{x}$ approaches the surface $\partial B$ from outside the nanowire.

\subsection{Evaluation of Various Integrals in (23) for $\ell>>b$}

The superscripts - and $(d)$ in $\bar{\phi}^{(d)},(d)$ in $\sigma^{(d)}$ and the subscript $d$ in $\epsilon_{d}$ are suppressed in this section in the interest of notational simplification. 


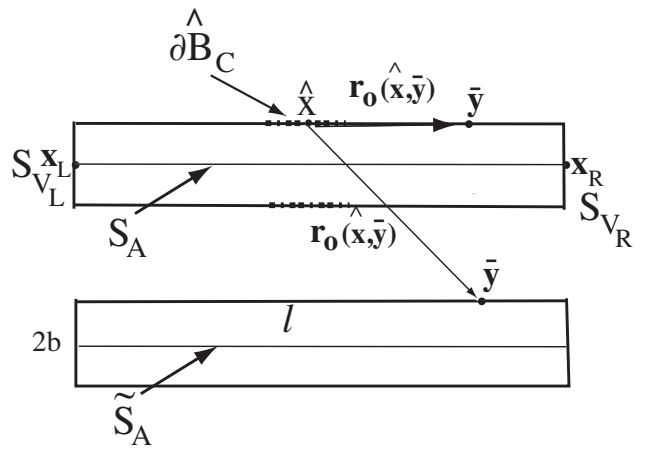

Figure 4: Nanowire and image

\subsubsection{First and second integrals in (23)}

Case (a) : $\mathbf{x}=\hat{\mathbf{x}} \in \partial \hat{B}_{C} \subset \partial B_{C}$ in Fig. 4. (This includes the top left and right corners of the upper wire).

These two integrals are written as $I_{1}$ and $I_{2}$, respectively.

The nonsingular second integral is approximated as [28]:

$$
I_{2}=\int_{\tilde{S}_{A}} \frac{q(\mathbf{y})}{4 \pi \epsilon r(\hat{\mathbf{x}}, \mathbf{y})} d \ell(\mathbf{y}), \quad \mathbf{x} \in \partial B_{C}
$$

where:

$$
q(\mathbf{y})=q(b, z)=2 \pi b \sigma(b, z)
$$

since $\sigma(\mathbf{y})$ is assumed to be axisymmetric.

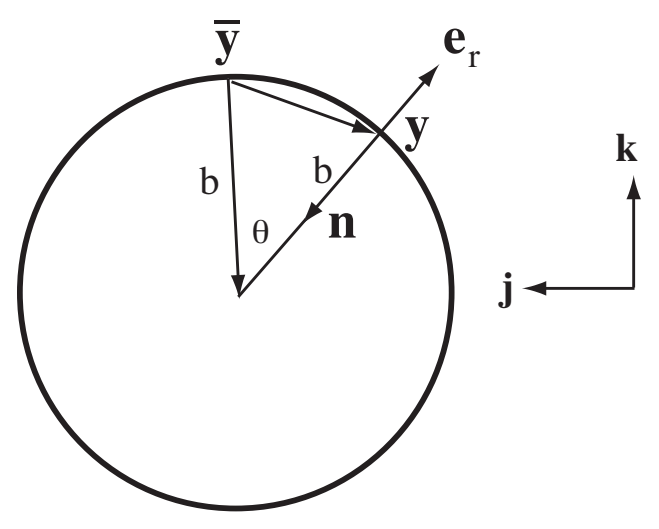

Figure 5: Geometry for evaluation of various integrals

The first integral above is decomposed as (see Fig. 5):

$$
I_{1}=\int_{S_{A}-\hat{S}_{A}} \frac{q(b, z)}{4 \pi \epsilon|z|} d z+\int_{\hat{S}_{A}} \int_{0}^{2 \pi} \frac{q(b, z) d \theta d z}{8 \pi^{2} \epsilon \sqrt{z^{2}+2 b^{2}(1-\cos (\theta))}}
$$

where (27) has been used. The source point $\mathbf{x} \in \partial \hat{\bar{B}}_{C}$ (with $\partial \hat{\bar{B}}_{C}$ is a line on $\partial B_{C}$ that corresponds 
to $\hat{S}_{A}$ ), while the field point $\mathbf{y} \in S_{A}$ (see Fig. 8 in [28]). Also, the length of the nanowire axis segment $\hat{S}_{A}$ is $\hat{\ell}$.

The second integral in (28) can be reduced to an elliptic integral (see Appendix A).

It is noted that if the axial coordinate of $\mathbf{x}$ is inside an element, then $\partial \hat{B}_{C}$ is that element. If the axial coordinate of $\mathbf{x}$ is on an edge of an element, then $\partial \hat{B}_{C}$ is the union of the element before and the element after the point $\mathbf{x}$.

Case (b) : $\mathbf{x}=\mathrm{x}_{L} \in S_{V L}$ or $\mathrm{x}_{R} \in S_{V R}$ on the axis of the upper wire (see Fig. 4).

First consider $\mathbf{x}=\mathbf{x}_{L}$. This time [28]:

$$
I_{1}+I_{2}=\int_{\partial \bar{B}_{C}} \frac{q(\mathbf{y})}{4 \pi r\left(\mathbf{x}_{L}, \mathbf{y}\right)} d \ell(\mathbf{y})+\int_{\partial \tilde{B}_{C}} \frac{q(\mathbf{y})}{4 \pi r\left(\mathbf{x}_{L}, \mathbf{y}\right)} d \ell(\mathbf{y})
$$

where $\partial \bar{B}_{C}$ is a line on $\partial B_{C}$ parallel to $S_{A}$.

The second integral in (29) is nonsingular and is evaluated as for (26). The first is nearly strongly singular. Its evaluation procedure is outlined in Section 2.5 of [28].

The case $\mathbf{x}=\mathbf{x}_{R}$ is analogous to $\mathbf{x}=\mathbf{x}_{L}$ and $I_{1}$ and $I_{2}$ for this case are evaluated in a manner similar to that outlined above.

\subsubsection{Third and fourth integrals in (23)}

Case (a) : $\mathbf{x}=\hat{\mathbf{x}} \in \partial \hat{B}_{C} \subset \partial B_{C}$ "far" from the ends $S_{V L}$ and $S_{V R}$ in Fig. 4.

One has:

$$
\begin{aligned}
I_{3}+I_{4} & =\int_{\partial B_{C}-\partial \hat{B}_{C}} \frac{\mathbf{r}(\hat{\mathbf{x}}, \mathbf{y}) \cdot \mathbf{n}(\mathbf{y})}{4 \pi r^{3}(\hat{\mathbf{x}}, \mathbf{y})} \phi(\mathbf{y}) d S(\mathbf{y})+\int_{\partial \hat{B}_{C}} \frac{\mathbf{r}(\hat{\mathbf{x}}, \mathbf{y}) \cdot \mathbf{n}(\mathbf{y})}{4 \pi r^{3}(\hat{\mathbf{x}}, \mathbf{y})}(\phi(\mathbf{y})-\phi(\hat{\mathbf{x}})) d S(\mathbf{y}) \\
& +\phi(\hat{\mathbf{x}}) \int_{\partial \hat{B}_{C}} \frac{\mathbf{r}(\hat{\mathbf{x}}, \mathbf{y}) \cdot \mathbf{n}(\mathbf{y})}{4 \pi r^{3}(\hat{\mathbf{x}}, \mathbf{y})} d S(\mathbf{y})+\int_{S_{V L} \cup S_{V R}} \frac{\mathbf{r}(\hat{\mathbf{x}}, \mathbf{y}) \cdot \mathbf{n}(\mathbf{y})}{4 \pi r^{3}(\hat{\mathbf{x}}, \mathbf{y})} \phi(r, \bar{z}) d S(\mathbf{y})
\end{aligned}
$$

where $\phi(\mathbf{y})=\phi(b, z)$ and $\bar{z}$ is the $z$ coordinate at $S_{V L}$ or $S_{V R}$. Also, $\partial \hat{B}_{C}$ is a ring of radius $b$, length $\hat{\ell}$.

1. The second integral in $(30)$ is weakly $(\mathcal{O}(1 / r))$ singular and computable (see Appendix A).

2. It is proved in Appendix B that the first (nonsingular) integral in (30) is $\mathcal{O}\left(b^{2}\right)$. It is assumed to vanish as $b \rightarrow 0$.

3. It is proved in Appendix B that the last (nonsingular) integral in (30) is $\mathcal{O}\left(b^{2}\right)$. It is assumed to vanish as $b \rightarrow 0$.

4. It is well known (see, for example, [13]) that the solid angle integral:

$$
\Omega(\partial S, \hat{\mathbf{x}})=\int_{\partial S} \frac{\mathbf{r}(\hat{\mathbf{x}}, \mathbf{y}) \cdot \mathbf{n}(\mathbf{y})}{r^{3}(\hat{\mathbf{x}}, \mathbf{y})} d S(\mathbf{y})=0
$$

where $\Omega$ is the solid angle subtended by any closed surface $\partial S$ at any point $\hat{\mathbf{x}}$ outside it.

One can interpret the third integral in (30) as:

$$
\phi(\hat{\mathbf{x}}) \int_{\partial S} \frac{\mathbf{r}(\hat{\mathbf{x}}, \mathbf{y}) \cdot \mathbf{n}(\mathbf{y})}{r^{3}(\hat{\mathbf{x}}, \mathbf{y})} d S(\mathbf{y})
$$


where $\partial S$ is the union of $\partial \hat{B}_{C}$ together with two flat disc closure surfaces. As noted before, the integrals on the flat discs vanish if $r_{0}>b$ and $b \rightarrow 0$. Therefore, this third integral in (30) vanishes.

Case (b) : $\mathbf{x}=\mathrm{x}_{L} \in S_{V L}$ or $\mathrm{x}_{R} \in S_{V R}$ on the axis of the upper wire (see Fig. 4).

This situation is discussed in Appendix C.

Case (c) : $\mathbf{x} \in S_{V L}$ or $\in S_{V R}$ at the upper left or right corner of the upper wire (see Fig. 15).

This situation is discussed in Appendix D.

\subsubsection{Last integral in (23)}

It is proved in Appendix B that the last integral in $(23)$ is $\mathcal{O}\left(b^{2}\right)$ and is assumed to vanish as $b \rightarrow 0$.

\subsection{Surviving Integrals from (23)}

The following quantities survive:

1. The first and second integrals (23)

2. The second integral in (30) with $\hat{\mathbf{x}} \in \partial \hat{B}_{C}$

3. The second integral in (30) with $\hat{\mathbf{x}}=\mathbf{x}_{L} \in S_{V_{L}}$. This integral approximately equals:

$$
\frac{1}{2}\left[\phi(0,0)-\phi(b, 0)-b \frac{\partial \phi}{\partial z}(b, 0)\right]
$$

4. The second integral in (30) with $\hat{\mathbf{x}}=\mathbf{x}_{R} \in S_{V_{R}}$. This integral approximately equals:

$$
\frac{1}{2}\left[\phi(0, \ell)-\phi(b, \ell)-b \frac{\partial \phi}{\partial z}(b, \ell)\right]
$$

\section{Coupling of FEM and BEM}

\subsection{Computational Domains}

The physical and image wires are shown in Fig. 6

The computational domain for the FEM is just the upper half of the upper (physical) wire (see, also, Fig. 3). Its image is the lower half of the lower (image) wire.

The computational domain for the BEM is the region outside the nanowires.

As mentioned before, the image tube is a nonphysical mathematical artifact which is used to guarantee zero potential on the ground plane without directly modeling it. This is done by relating values of potential and charge on the image wire, with those in the physical wire, as shown in Fig. 6. The FEM problem is nonlinear and, in general, the FEM equations are not satisfied inside the image nanowire. 


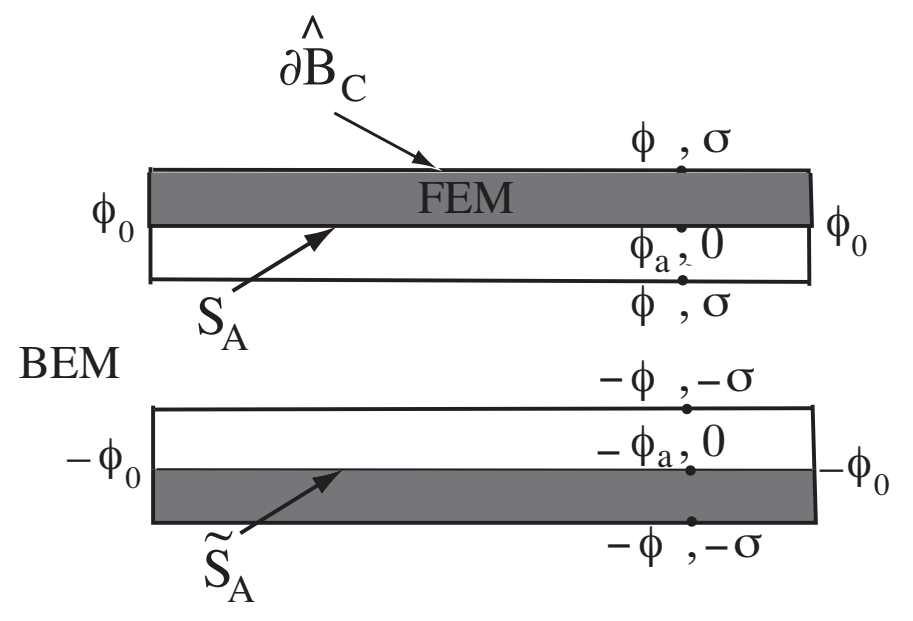

Figure 6: BEM and FEM domains

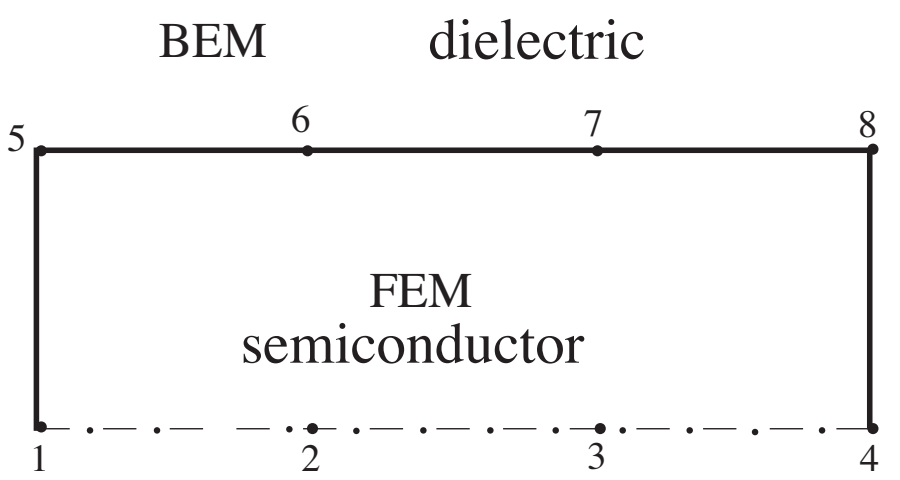

Figure 7: Numerical implementation of FEM/BEM

\subsection{Boundary Conditions, Unknowns and Equations}

A single finite element model for the upper wire is shown, for simplicity, in Fig. 7. Two schemes for solving the coupled problem are shown in Tables 1 and 2, respectively. In both cases, $\phi=0$ is imposed on the ends of the wire, $\partial \phi / \partial r=0$ along its axis, and transition conditions at nodes 6 and 7 . Since $\phi$ is assumed to be constant along the edges, $\partial \phi / \partial r^{(s)}=0$ at nodes 5 and 8 as well. Also, the FEM equations are used at all 8 nodes.

In the first scheme (Table 1) the axial derivatives are unknown at the end nodes and these are allowed to be discontinuous. Finally, the BEM equations are imposed at nodes 1,4,8,7,6 and 5 and hypersingular BEM (HBEM) equations must be imposed at 5,8. (This is a consequence of a wellknown problem - shortage of equations when the standard BEM is imposed at Dirichlet corners). One has 16 unknowns and an equal number of equations.

The simplified second scheme (Table 2) uses the fact that a nanowire is very thin. This time, only the internal axial derivatives are considered at the end nodes and the BEM equations are imposed at nodes $8,7,6,5$. (As seen in (23), certain integrals on the ends of the nanowire are neglected, as was done before in [28]). This scheme has 12 equations and an equal number of 


\begin{tabular}{|c|c|c|}
\hline boundary conditions & unknowns & equations \\
\hline$\phi=0: 1,4,8,5$ & $\phi: 2,3,7,6$ & FEM $: 1,2,3,4,8,7,6,5$ \\
$\frac{\partial \phi}{\partial r}^{(s)}=0: 1,2,3,4,8^{\star}, 5^{\star}$ & $\frac{\partial \phi}{\partial r}^{(d)}: 8,7,6,5$ & BEM $: 1,4,8,7,6,5$ \\
$\epsilon^{(s)} \frac{\partial \phi}{\partial r}^{(s)}=\epsilon^{(d)} \frac{\partial \phi}{\partial r}^{(d)}: 7,6$ & $\frac{\partial \phi}{\partial z}^{(s)}: 1,4,8,5$ & HBEM $: 5,8$ \\
& $\frac{\partial \phi}{\partial z}^{(d)}: 1,4,8,5$ & \\
\hline
\end{tabular}

Table 1: Scheme one : boundary conditions, unknowns and equations (see Fig. 7). : These conditions are derived from $\phi=$ constant at the ends

\begin{tabular}{|c|c|c|}
\hline boundary conditions & unknowns & equations \\
\hline$\phi=0: 1,4,8,5$ & $\phi: 2,3,7,6$ & FEM : $1,2,3,4,8,7,6,5$ \\
$\frac{\partial \phi}{\partial r}^{(s)}=0: 1,2,3,4,8^{\star}, 5^{\star}$ & $\frac{\partial \phi}{\partial r}^{(d)}: 8,7,6,5$ & BEM : 8,7,6,5 \\
$\epsilon^{(s)} \frac{\partial \phi}{\partial r}{ }^{(s)}=\epsilon^{(d)} \frac{\partial \phi}{\partial r}^{(d)}: 7,6$ & $\frac{\partial \phi}{\partial z}^{(s)}: 1,4,8,5$ & \\
\hline
\end{tabular}

Table 2: Scheme two : boundary conditions, unknowns and equations (see Fig. 7). $\star$ : These conditions are derived from $\phi=$ constant at the ends

unknowns.

The FEM option two (see Section 3.1.3) as well as scheme two are used to obtain the numerical results that are presented later in this paper.

\subsection{Coupling Strategy and Stopping Criteria}

Consider Fig. 7 but with many elements instead of a single one. The potential $\phi$ is prescribed at the corners, $\partial \phi / \partial r=0$ on the rest of the symmetry axis, and

$$
\epsilon^{(s)} \frac{\partial \phi}{\partial r}^{(s)}=\epsilon^{(d)} \frac{\partial \phi}{\partial r}^{(d)}
$$

on the rest of the upper line.

The proposed iterative algorithm is as follows:

1. Guess $\phi$ on the rest of the upper line.

2. Solve the BEM equations for $\partial \phi / \partial r^{(d)}$ on the upper line.

3. Find $\partial \phi / \partial r^{(s)}$ on the upper line (except at its ends) from (33).

4. Solve the FEM equations in the semiconductor with prescribed $\phi$ at the corners, $\partial \phi / \partial r=0$ on the rest of the axis of symmetry, and $\partial \phi / \partial r^{(s)}$ from step (3) above on the rest of the upper line. Determine $\phi$ on the entire boundary of the body and $\partial \phi / \partial z^{(s)}$ at its ends.

5. Go to step (2) and iterate until convergence.

The FEM residue $[r]$ is defined as:

$$
[r]=[K][\phi]-[F]
$$

The iterations stop when both of the following criteria are met. 
1.

$$
\sqrt{\frac{\sum_{i=1}^{N}\left[\phi_{\text {new }}(i)-\phi_{\text {old }}(i)\right]^{2}}{N}} \leq \epsilon_{1}
$$

2 .

$$
\sqrt{\frac{\sum_{i=1}^{N}\left[r_{n e w}(i)\right]^{2}}{N}} \leq \epsilon_{2}
$$

The values of the tolerances used are $\epsilon_{1}=10^{-5}$ and $\epsilon_{2}=0.6 \times 10^{-5}$

\section{$6 \quad$ Numerical Results}

\subsection{Values of Material and Geometrical Parameters}

Material parameters used in the semiconductor simulations reported below are:

$\epsilon_{d}=8.854 \times 10^{-12} \mathrm{~F} / \mathrm{m}$

$\epsilon_{s}=11.7 \epsilon_{d}$

$e=1.6 \times 10^{-19} \mathrm{C}$

$N_{D}=10^{18} \mathrm{~cm}^{-3}$

$N_{A}=0$

$n_{i}=3.1 \times 10^{16} T^{3 / 2} \exp \left[-\frac{0.966 \times 10^{-19}}{k_{B} T}\right] \mathrm{cm}^{-3}$

$k_{B}=1.38 \times 10^{-23} \mathrm{~J} / \mathrm{K}$

The temperature $T=300 \mathrm{~K}$ and the potentials on the left and right sides of the nanowire (see Fig. 6) are $\phi_{0}=0$.

The nominal values of the geometrical parameters for the semiconductor calculations are: $\ell=1000 \mathrm{~nm}, b=1 \mathrm{~nm}[37]$ and $g=500 \mathrm{~nm}$.

\subsection{Code Verification}

\subsubsection{FEM code}

The FEM equations are used to solve the nonlinear Poisson equation (1) in the axisymmetric silicon nanowire. The chosen boundary conditions are (see Fig. 6) $\phi_{0}=0$ and $\partial \phi / \partial r=0$ on the upper line of the upper wire. The material parameters and temperature are as given above, and the geometrical parameters used are $: \ell=1000 \mathrm{~nm}$ and $b=1 \mathrm{~nm}$. The converged numerical solutions at the center of the upper line (with 135 finite elements) are $\phi=1.2451 \times 10^{-2} \mathrm{~V}$ from the present code and the same value (to five significant digits) from [38].

\subsubsection{BEM code}

The BEM code is verified by solving for the charge distribution on a silicon conductor. (Conducting carbon nanotubes were considered in [28]). The (uniform) potential on the conductor is chosen to be $1 \mathrm{~V}$. The dimensions of the conductor are : $\ell=3000 \mathrm{~nm}, b=1 \mathrm{~nm}$, and $g=500 \mathrm{~nm}$. The charge density at the mid-point of the conductor, from the BEM code with 425 boundary elements, is $8.145 \mathrm{pC} / \mathrm{m}$, while that from Table II of $[28]$ is $8.15 \mathrm{pC} / \mathrm{m}$. 


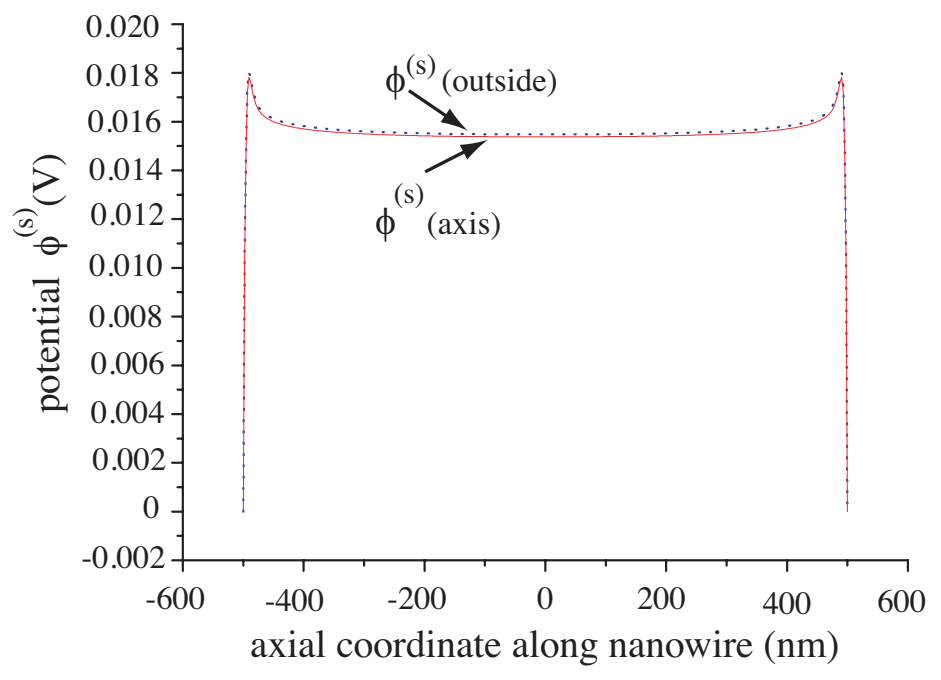

Figure 8: Potential $\phi^{(s)}$ along nanowire $(\ell=1000 \mathrm{~nm}, g=500 \mathrm{~nm}, b=1 \mathrm{~nm})$

\subsubsection{Coupled code}

This situation is discussed below.

\subsection{Numerical Examples}

The coupled problem is first solved for the baseline case with $\ell=1000 \mathrm{~nm}, b=1 \mathrm{~nm}$ and $g=500$ $\mathrm{nm}$. Converged results for the potential $\phi^{(s)}$ and charge density $\rho^{(s)}$ along the nanowire are shown in Figs. 8 and 9, respectively. A total of 142 elements are used along the wire. Corresponding results for thin conducting tubes, which have constant potential and charge distributions only on their surfaces, are available in [28].

The above result has been verified by separately computing the left and right hand sides of (1). The Laplacian of the potential is computed by using the appropriate interpolation functions for the potential, while the right hand side is computed directly. The results, at the mid point of the upper line on the upper wire, are 0.0419 and $0.0431 \mathrm{C} / \mathrm{cm}^{3}$, respectively.

Numerical results have also been computed for different values of length $(\ell=500,1500 \mathrm{~nm}$; with $g=500$ and $b=1 \mathrm{~nm}$ ). Results for $\ell=1500 \mathrm{~nm}$ (with 214 elements) are shown in Figs. 10 and 11, respectively. The potential and charge distributions are qualitatively very similar to those in Figs. 8 and 9, with a suitable stretching of the $x$ axis.

Numerical results for $\ell=1000, b=1 \mathrm{~nm}$, with different values of the gap $(g=300,700 \mathrm{~nm})$, are substantially the same as the baseline results in Figs. 8 and 9 - i.e. they are insensitive to the value of the gap. Of course, these results would change for small values of $g$ (for example of the order of $10 \mathrm{~nm}$ ), but such results for the potential and charge would no longer be axisymmetric and the mathematical formulation employed in this paper would break down. 


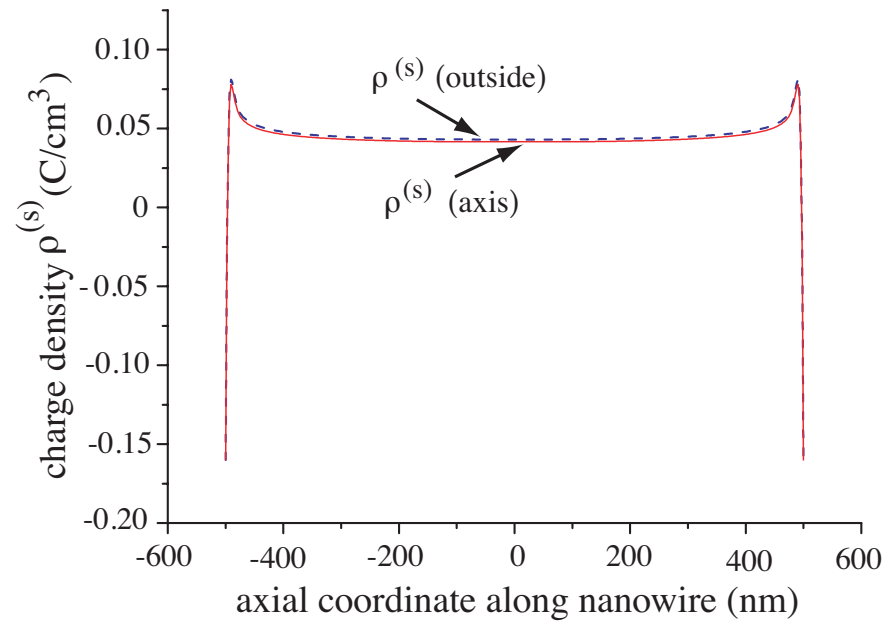

Figure 9: Charge density $\rho^{(s)}$ along nanowire $(\ell=1000 \mathrm{~nm}, g=500 \mathrm{~nm}, b=1 \mathrm{~nm})$

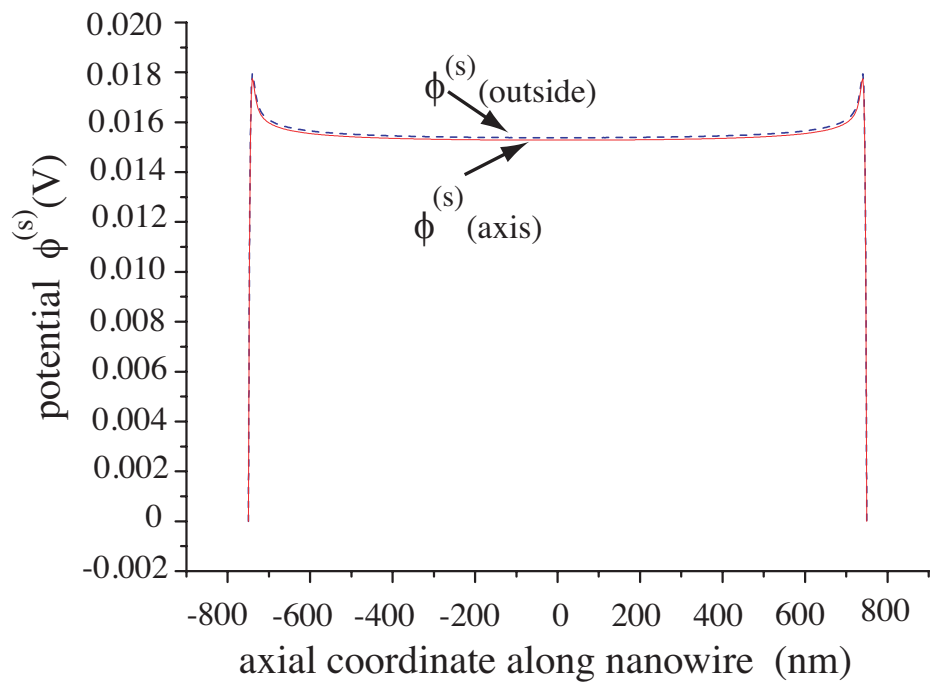

Figure 10: Potential $\phi^{(s)}$ along nanowire $(\ell=1500 \mathrm{~nm}, g=500 \mathrm{~nm}, b=1 \mathrm{~nm})$ 


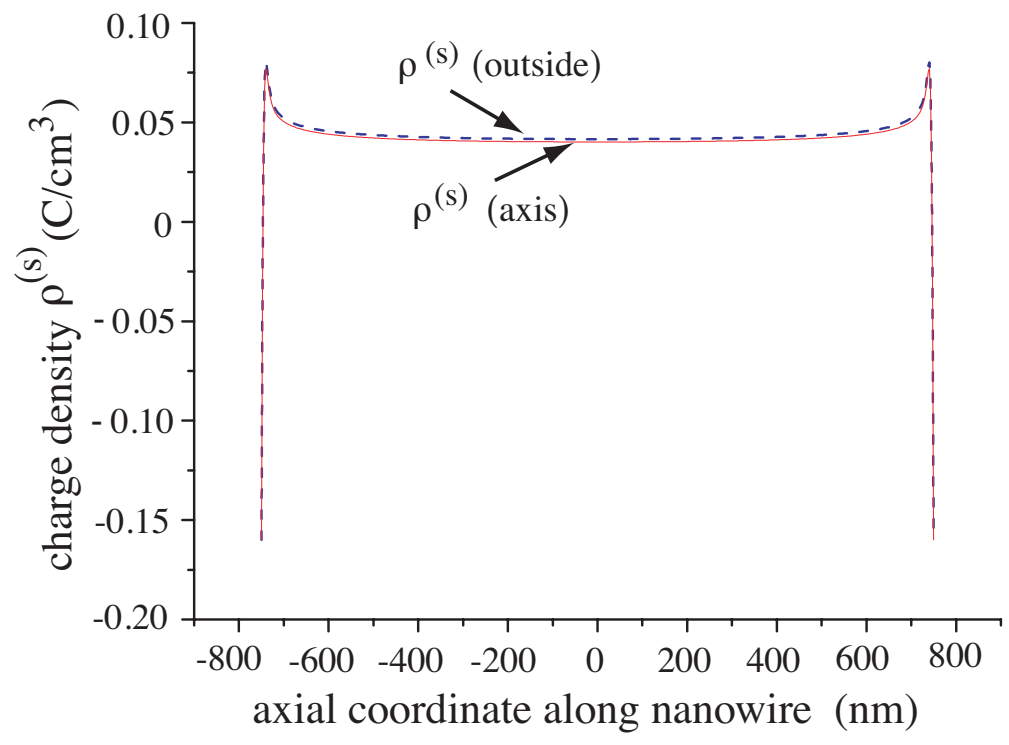

Figure 11: Charge density $\rho^{(s)}$ along nanowire $(\ell=1500 \mathrm{~nm}, g=500 \mathrm{~nm}, b=1 \mathrm{~nm})$

\section{Concluding Remarks}

A BEM approach for the electrostatic modelling of the 3-D region exterior to a conducting carbon nanotube, taking advantage of the typical large aspect ratio of such a tube, has been presented in [28]. The present paper presents a similar analysis for a semiconducting silicon nanowire. A hybrid semiclassical (Laplace/Poisson) model is adopted in the semiconductor, and the potential and charge distribution along the wire are determined by a coupled FEM/BEM approach. The present approach overcomes the problem of dealing with nearly singular matrices that typically occur when using the BEM for thin structures. It is also very efficient.

A limitation of the present work is the assumption that the potential and charge distribution inside the nanowire is axisymmetric. This is true provided that the gap between a nanowire and the ground is large enough (at least around ten to twenty times the diameter of the nanowire). It has also been observed that a very fine BEM mesh can cause small oscillations (as a function of the axial variable) in the calculated potential and charge about its mean value. It is felt that this is due to the assumption $\ell>>b$ starting to break down as the length of a boundary element becomes small. Such oscillations are absent in the results presented in this paper.

For small diameter nanowires, quantum effects can become dominant and the semi-classical model presented above needs to be corrected (see, e.g. [26, 27] for 2-D analysis of this problem). As mentioned above, the primary focus of the present paper is to develop an efficient computational framework and the semi-classical model has been considered as an example. Quantum effects will be included in the future.

Electromechanical coupling to include deformation of the nanowire will also be considered in future research. This, together with inclusion of inertia forces, will enable the modeling and solution of electrodynamic problems. (See, e.g. [24] for dynamic analysis of MEMS).

\section{Acknowledgements}

This research has been supported by Grant \#s EEC-0303674 and CMS-0508466 of the National Science Foundation to Cornell University. 


\section{Appendix A : Second integral in (28) and second integral in (30)}

\section{Definitions of elliptic integrals}

Elliptic integrals of the first and third kinds are used in this section. These are defined as:

$$
\begin{gathered}
E_{!}(\phi, k)=\int_{0}^{\phi} \frac{d \theta}{\sqrt{1-k^{2} \sin ^{2}(\theta)}} \\
E_{3}(n, \phi, k)=\int_{0}^{\phi} \frac{d \theta}{\left(1-n \sin ^{2}(\theta)\right) \sqrt{1-k^{2} \sin ^{2}(\theta)}}
\end{gathered}
$$

Second integral in (28)

$$
J=\frac{1}{8 \pi^{2} \epsilon} \int_{\hat{S}_{A}} q(b, z) d z \int_{0}^{2 \pi} \frac{d \theta}{\sqrt{z^{2}+4 b^{2} \sin ^{2}(\theta / 2)}}
$$

The inner integral in (A-3) can be written as:

$$
\frac{4}{\sqrt{z^{2}+4 b^{2}}} E_{1}\left(\frac{\pi}{2}, \frac{2 b}{\sqrt{z^{2}+4 b^{2}}}\right)
$$

where $E_{1}$ denotes the elliptic integral of the first kind.

\section{Second integral in (30) for case (a)}

From Figs. 4 and 5, one has:

$$
\begin{aligned}
\mathbf{r}(\hat{\mathbf{x}}, \mathbf{y}) & =\mathbf{r}_{\mathbf{0}}(\hat{\mathbf{x}}, \overline{\mathbf{y}})-b \mathbf{k}+b \mathbf{e}_{r}, \quad \mathbf{r} \cdot \mathbf{n}=-\mathbf{r} \cdot \mathbf{e}_{r}=-\mathbf{r}_{0} \cdot \mathbf{e}_{r}+b \cos (\theta)-b=b(\cos (\theta)-1) \\
r^{2} & =z^{2}+2 b^{2}(1-\cos (\theta))
\end{aligned}
$$

Therefore, this integral can be written as:

$$
S_{2}=-\int_{\partial \hat{\bar{B}}_{C}}[\phi(\mathbf{y})-\phi(\hat{\mathbf{x}})] d z \int_{0}^{2 \pi} \frac{4 b^{2} \sin ^{2}(\theta / 2)}{8 \pi\left[z^{2}+4 b^{2} \sin ^{2}(\theta / 2)\right]^{3 / 2}} d \theta
$$

The inner integral in (A-6) can be written in terms of elliptic integrals as follows:

$$
\frac{1}{2 \pi \sqrt{z^{2}+4 b^{2}}} E_{1}\left(\frac{\pi}{2}, \frac{2 b}{\sqrt{z^{2}+4 b^{2}}}\right)-\frac{z^{2}}{2 \pi\left[z^{2}+4 b^{2}\right]^{3 / 2}} E_{3}\left[\frac{4 b^{2}}{z^{2}+4 b^{2}}, \frac{\pi}{2}, \frac{2 b}{\sqrt{z^{2}+4 b^{2}}}\right]
$$

where $E_{1}$ and $E_{3}$ denote elliptic integrals of the first and third kinds, respectively. 


\section{Appendix B : First and last integrals in (30) and the last integral in $(23)$}

First integral in (30) for case (a)

As before, from Figs. 4 and 5, one has:

$$
\mathbf{r}(\hat{\mathbf{x}}, \mathbf{y})=\mathbf{r}_{\mathbf{0}}(\hat{\mathbf{x}}, \overline{\mathbf{y}})-b \mathbf{k}+b \mathbf{e}_{r}, \quad \mathbf{r} \cdot \mathbf{n}=-\mathbf{r} \cdot \mathbf{e}_{r}=-\mathbf{r}_{0} \cdot \mathbf{e}_{r}+b \cos (\theta)-b=b(\cos (\theta)-1)
$$

Assuming $r_{0}>>b$, and one can write:

$$
b \int_{0}^{2 \pi} \frac{\mathbf{r}(\hat{\mathbf{x}}, \mathbf{y}) \cdot \mathbf{n}(\mathbf{y})}{4 \pi r^{3}(\hat{\mathbf{x}}, \mathbf{y})} d \theta \approx b \int_{0}^{2 \pi} \frac{\mathbf{r}(\hat{\mathbf{x}}, \mathbf{y}) \cdot \mathbf{n}(\mathbf{y})}{4 \pi r_{0}^{3}(\hat{\mathbf{x}}, \overline{\mathbf{y}})} d \theta=-\frac{b^{2}}{2 r_{0}^{3}}
$$

It is noted that, by assumption, $\phi(\mathbf{y})$ is axisymmetric. Therefore, the first integral in (30) is $\mathcal{O}\left(b^{2}\right)$ and is assumed to vanish as $b \rightarrow 0$.

\section{Last integral in (23)}

This integral is very similar to the first integral in (30), except that (see Fig. 4) $\overline{\mathbf{y}}$ now lies on the image nanowire $\partial \tilde{B}_{C}$. A calculation very similar to the one in the preceding section shows that this integral is also $\mathcal{O}\left(b^{2}\right)$ and is assumed to vanish as $b \rightarrow 0$.

\section{Last integral in (30) for case (a)}

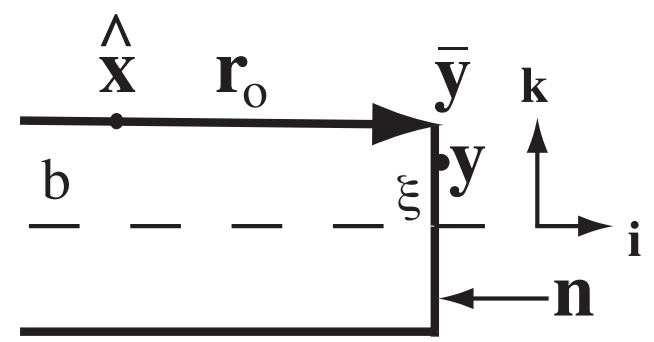

Figure 12: Integration over right cap

First consider the integral over the right cap $S_{V_{R}}$. From Fig. 12:

$$
\mathbf{r}(\hat{\mathbf{x}}, \mathbf{y})=r_{0}(\hat{\mathbf{x}}, \overline{\mathbf{y}}) \mathbf{i}-(b-\xi) \mathbf{k}
$$

With $\mathbf{n}=-\mathbf{i}, \mathbf{r} \cdot \mathbf{n}=-r_{0}$. Assuming $r_{o}>>b$, one has:

$$
\frac{\mathbf{r} \cdot \mathbf{n}}{r^{3}}=-\frac{r_{0}}{\left[r_{0}^{2}+(b-\xi)^{2}\right]^{3 / 2}} \approx-\frac{1}{r_{0}^{2}}
$$

Therefore, the last integral in (30) over the right cap becomes:

$$
-\int_{\xi=0}^{b} \frac{\phi\left(\xi, z_{R}\right) 2 \pi \xi d \xi}{4 \pi r_{0}^{2}}
$$

With $\phi \approx \mathcal{O}(1)$, the above integral is $\mathcal{O}\left(b^{2}\right)$, and is assumed to vanish as $b \rightarrow 0$.

It can be shown in an analogous manner that this integral over the left cap $S_{V_{L}}$ is also $\mathcal{O}\left(b^{2}\right)$. 


\section{Appendix C : Equation (30) for case (b) with $\hat{\mathbf{x}} \rightarrow \mathrm{x}_{L} \in S_{V_{L}}$}

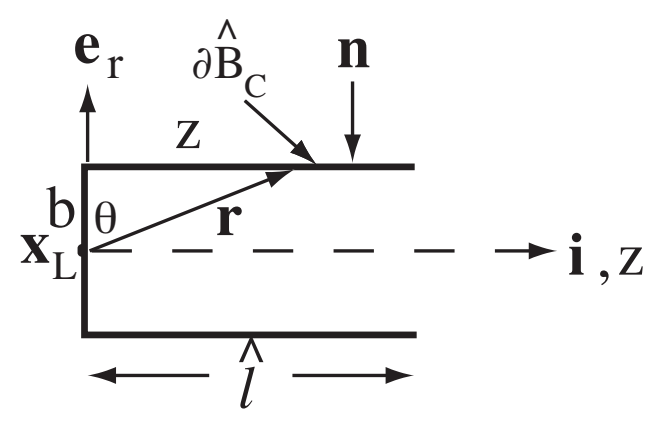

Figure 13: Integration over curved surface

Start with (30) with $\hat{\mathbf{x}} \rightarrow \mathbf{x}_{L}$ and see Figs 4 and 13 .

1. The first integral in (30) is $\mathcal{O}\left(b^{2}\right)$ and is assumed to vanish as $b \rightarrow 0$.

2. For the second and third integrals $\left(J_{2}\right.$ and $\left.J_{3}\right)$ in (30) (see Fig. 13):

$$
\mathbf{r}\left(\mathbf{x}_{\mathbf{L}}, \mathbf{y}\right)=b \mathbf{e}_{r}+z \mathbf{i}
$$

where $\mathbf{e}_{r}$ is the radial unit vector at $\mathbf{x}_{L}\left(\mathbf{e}_{r}=-\mathbf{n}(\mathbf{y})\right)$ and $\mathbf{i}$ is the unit axial vector. Hence, $\mathbf{r} \cdot \mathbf{n}=-b$.

First consider the integral $J_{2}$. Referring to (20), one has:

$$
\phi(\mathbf{y})-\phi\left(\mathbf{x}_{L}\right)=[\phi(b, z)-\phi(b, 0)]+[\phi(b, 0)-\phi(0,0)]=z \frac{\partial \phi}{\partial z}(b, 0)+\phi(b, 0)-\phi(0,0)
$$

Therefore:

$$
J_{2}=-\int_{0}^{\hat{\ell}} \frac{b^{2}[z \partial \phi / \partial z(b, 0)+\phi(b, 0)-\phi(0,0)]}{2\left[b^{2}+z^{2}\right]^{3 / 2}} d z
$$

With $z=b \tan (\theta)$ and $\tan (\alpha)=\hat{\ell} / b$, one gets:

$$
J_{2}=-\frac{b(1-\cos (\alpha))}{2} \frac{\partial \phi}{\partial z}(b, 0)+\frac{\sin (\alpha)}{2}[\phi(0,0)-\phi(b, 0)]
$$

As $b \rightarrow 0, \alpha \rightarrow \pi / 2$ and $J_{2} \rightarrow(1 / 2)[\phi(0,0)-\phi(b, 0)-b \partial \phi / \partial z(b, 0)]$

Next:

$$
J_{3}=-\phi\left(\mathbf{x}_{L}\right) \int_{\partial \hat{B}_{C}} \frac{b}{4 \pi r^{3}\left(\mathbf{x}_{L}, \mathbf{y}\right)} d S(\mathbf{y})=-\phi\left(\mathbf{x}_{L}\right) \int_{0}^{\hat{l}} \frac{2 \pi b^{2}}{4 \pi\left(b^{2}+z^{2}\right)^{3 / 2}} d z=-\phi\left(\mathbf{x}_{L}\right) \sin (\alpha) / 2
$$

where $\sin (\alpha)=\hat{\ell} / \sqrt{b^{2}+\hat{\ell}^{2}}$.

As $b \rightarrow 0, \sin (\alpha) \rightarrow 1$ and one has $J_{3}=-\phi\left(\mathbf{x}_{L}\right) / 2$. 


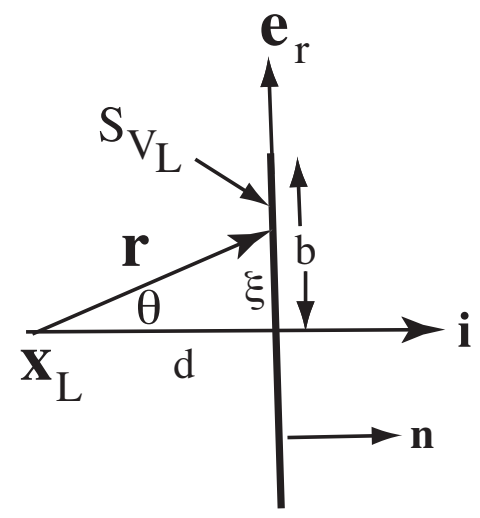

Figure 14: Integration over left cap

3. The fourth integral in (30).

First consider only the integral over $S_{V L}$ (see Fig. 14). This integral is evaluated in a finite part sense [36]. Therefore, the source point $\mathbf{x}_{L}$ is first chosen to lie at a distance $d$ from $S_{V_{L}}$, and then the limit $d \rightarrow 0$ is taken.

$$
\begin{aligned}
J_{4} & =\int_{S_{V L}} \frac{\mathbf{r}\left(\mathbf{x}_{L}, \mathbf{y}\right) \cdot \mathbf{n}(\mathbf{y})}{4 \pi r^{3}\left(\mathbf{x}_{L}, \mathbf{y}\right)} \phi(\mathbf{y}) d S(\mathbf{y})=\int_{S_{V L}} \frac{\mathbf{r}\left(\mathbf{x}_{L}, \mathbf{y}\right) \cdot \mathbf{n}(\mathbf{y})}{4 \pi r^{3}\left(\mathbf{x}_{L}, \mathbf{y}\right)}\left[\phi(\mathbf{y})-\phi\left(\mathbf{x}_{L}\right)\right] d S(\mathbf{y}) \\
& +\phi\left(\mathbf{x}_{L}\right) \int_{S_{V L}} \frac{\mathbf{r}\left(\mathbf{x}_{L}, \mathbf{y}\right) \cdot \mathbf{n}(\mathbf{y})}{4 \pi r^{3}\left(\mathbf{x}_{L}, \mathbf{y}\right)} d S(\mathbf{y}) \equiv J_{41}+J_{42}
\end{aligned}
$$

The first and second integrals, $J_{41}$ and $J_{42}$ above, have (see Fig. 13):

$$
\mathbf{r}=d \mathbf{i}+\xi \mathbf{e}_{r}, \quad \mathbf{r} \cdot \mathbf{n}=\mathbf{r} \cdot \mathbf{i}=d
$$

Consider, first the integral $J_{41}$. One gets (see (20) with $r$ replaced by $\xi$ ):

$$
\begin{gathered}
\phi(\mathbf{y})-\phi\left(\mathbf{x}_{L}\right)=\left(\xi^{2} / b^{2}\right)[\phi(b, 0)-\phi(0,0)] \\
J_{41}=\frac{d}{2 b^{2}}[\phi(b, 0)-\phi(0,0)] \int_{0}^{b} \frac{\xi^{3}}{\left(d^{2}+\xi^{2}\right)^{3 / 2}} d \xi
\end{gathered}
$$

With $\xi=d \tan (\theta)$, the integral in (A-21) becomes:

$$
d \int_{0}^{\beta} \frac{\sin ^{3}(\theta)}{\cos ^{2}(\theta)} d \theta=d\left[\sin ^{4}(\beta) \sec (\beta)+\cos ^{3} \beta-3 \cos (\beta)+2\right]
$$

where $\cos (\beta)=d / \sqrt{\left(d^{2}+b^{2}\right)}$.

Therefore, as $d \rightarrow 0, J_{41} \rightarrow 0$.

Next:

$$
J_{42}=\phi\left(\mathbf{x}_{L}\right) \int_{0}^{b} \frac{2 d \pi \xi d \xi}{4 \pi\left[d^{2}+\xi^{2}\right]^{3 / 2}}=\frac{\phi\left(\mathbf{x}_{L}\right)}{2}[1-\cos (\beta)]
$$


where, as before. $\cos (\beta)=d / \sqrt{\left(d^{2}+b^{2}\right)}$.

As $d \rightarrow 0, \beta \rightarrow \pi / 2$ and $J_{42}=\phi\left(\mathbf{x}_{L}\right) / 2$. Therefore, $J_{3}+J_{42}=0$.

The integral $J_{4}$, evaluated on $S_{V R}$, is $\mathcal{O}\left(b^{2}\right)$, and vanishes as $b \rightarrow 0$.

A very similar discussion applies to (30) when $\hat{\mathbf{x}} \rightarrow \mathbf{x}_{R} \in S_{V_{R}}$. This time, the surviving term is: $(1 / 2)[\phi(0, \ell)-\phi(b, \ell)-b \partial \phi / \partial z(b, \ell)]$.

\section{Appendix D : Equation (30) for case (c) with $\mathrm{x}$ at top left and right corners (Figure 15)}

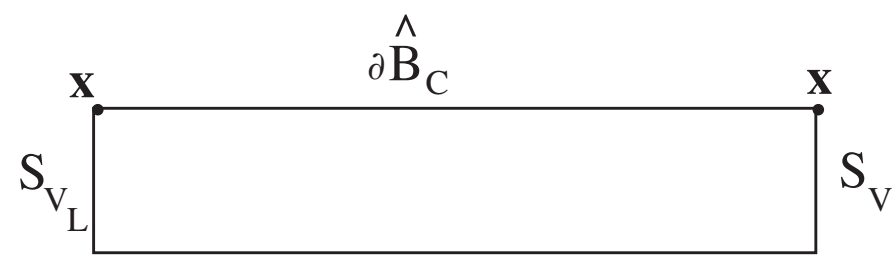

Figure 15: Solid angle integral

Consider, first. $\mathbf{x}$ at the top left corner. In (30), the first two integrals are the same as in case (a) in Section 4.2.2. The last two integrals in (30) add up to zero as shown below.

Proof of $J_{3}+J_{4}=0$ for case (c). It is interesting to note that the solid angle integral (see $(31))$ :

$$
\int_{\partial D} \frac{\mathbf{r}(\mathbf{x}, \mathbf{y}) \cdot \mathbf{n}(\mathbf{y})}{4 \pi r^{3}(\mathbf{x}, \mathbf{y})} d S(\mathbf{y})=0
$$

where $\partial D$ is any closed region and $\mathbf{x} \rightarrow \partial D$ from the outside. One can choose $\partial D=\partial \hat{B}_{C} \cup S_{V_{L}} \cup S_{V}$ (see Fig. 15). With the integral in (A-24) over $S_{V}$ vanishing for $b \rightarrow 0$, one has:

$$
\int_{\partial \hat{B}_{C} \cup S_{V_{L}}} \frac{\mathbf{r}(\mathbf{x}, \mathbf{y}) \cdot \mathbf{n}(\mathbf{y})}{4 \pi r^{3}(\mathbf{x}, \mathbf{y})} d S(\mathbf{y})=0
$$

Therefore, $J_{3}+J_{42}=0$ also applies for a collocation point $\mathbf{x}$ at the top left corner in Fig. 15 . Finally, with $J_{41}=0$, one has $J_{3}+J_{4}=0$ for this case.

It can be shown that the results for $\mathbf{x}$ at the upper right corner are the same as those for $\mathbf{x}$ at the upper left corner. 


\section{References}

[1] Roukes ML. Nanoelectromechanical Systems. Solid-State Sensor and Actuator Workshop. Hilton Head, SC, 2000.

[2] Davis ZJ, Abadal G, Kuhn O, Hansen O, Grey F, Boisen A. Fabrication and characterization of nanoresonating devices for mass detection. Journal of Vacuum Science Technology B 2000; 18:612-616.

[3] Craighead HG. Nanoelectromechanical systems. Science 2000; 290:1532-1535.

[4] Cui Y, Wei Q, Park H, Lieber CM. Nanowire nanosensors for highly sensitive and selective detection of biological and chemical species. Science 2001; 293:1289-1292.

[5] Yang J, Ono T, Esashi M. Surface effects and high quality factors in ultrathin single-crystal silicon cantilevers. Applied Physics Letters 2000; 77:3860-3862.

[6] Carr DW, Evoy S, Sekaric L, Craighead HG, Parpia JM. Measurement of mechanical resonance and losses in nanometer scale silicon wires. Applied Physics Letters 1999; 75:920-922.

[7] Boggild P, Hansen TM, Tanasa T, Grey F. Fabrication and actuation of customized nanotweezers with a $25 \mathrm{~nm}$ gap. Nanotechnology 2001; 12:331-335.

[8] Cleland AL, Roukes ML. Fabrication of high frequency nanometer scale mechanical resonators from bulk Si crystals. Applied Physics Letters 1996; 69:2653-2655.

[9] Mukherjee S. Boundary Element Methods in Creep and Fracture. Applied Science Publishers: London, 1982.

[10] Banerjee PK. The Boundary Element Methods in Engineering. McGraw Hill Europe: Maidenhead, Berkshire, England, 1994.

[11] Chandra A, Mukherjee S. Boundary Element Methods in Manufacturing. Oxford University Press: New York, 1997.

[12] Bonnet M. Boundary Integral Equation Methods for Solids and Fluids. Wiley: Chichester, UK, 1999.

[13] Mukherjee S, Mukherjee, YX. Boundary Methods: Elements Contours and Nodes. Taylor and Francis, CRC Press: Boca Raton, FL, 2005.

[14] Yang TY. Finite Element Structural Analysis. Prentice-Hall: New Jersey, 1986.

[15] Zienkiewicz OC, Taylor RL. The Finite Element Method, Vols. 1,2, 4th. ed. McGraw Hill: Maidenhead, Berkshire, UK, 1994.

[16] Hughes TJR. The Finite Element Method : Linear Static and Dynamic Finite Element Analysis. Dover: Mineola, NY, 2000.

[17] Senturia SD, Harris RM, Johnson BP, Kim S, Nabors K, Shulman MA, White JK. A computer-aided design system for microelectromechanical systems (MEMCAD). Journal of Micro-Electro-Mechanical Systems 1992; 1:3-13. 
[18] Nabors K, White J. FastCap: a multi-pole accelerated 3-D capacitance extraction program. IEEE Transactions on Computer Aided Design and Integrated Circuits and Systems 1991; 10:1447-1459.

[19] Gilbert JR, Legtenberg R, Senturia SD. 3D coupled electromechanics for MEMS : applications of CoSolve-EM. Proceedings IEEE MEMS 1995; 122-127.

[20] Shi F, Ramesh P, Mukherjee S. Simulation methods for micro-electro-mechanical structures (MEMS) with application to a microtweezer. Computers and Structures 1995; 56:769-783.

[21] Aluru NR, White J. An efficient numerical technique for electromechanical simulation of complicated microelectromechanical structures. Sensors and Actuators A 1997; 58:1-11.

[22] Mukherjee S, Bao Z, Roman M, Aubry N. Nonlinear mechanics of MEMS plates with a total Lagrangian approach. Computers and Structures 2005; 83:758-768.

[23] Shi F, Ramesh P, Mukherjee S. Dynamic analysis of micro-electro-mechanical systems. International Journal for Numerical Methods in Engineering 1996; 39:4119-4139.

[24] De SK, Aluru NR. Full-Lagrangian schemes for dynamic analysis of electrostatic MEMS. Journal of Microelectromechanical Systems 2004; 13:737-758.

[25] Ke C, Espinosa HD. Numerical analysis of nanotube-based NEMS devices - Part I: Electrostatic charge distribution on multiwalled nanotubes. ASME Journal of Applied Mechanics 2005; 72:721-725.

[26] Li G, Aluru NR. Hybrid techniques for electrostatic analysis of nanoelectromechanical systems. Journal of Applied Physics 2004; 96:2221-2231.

[27] Tang Z, Xu Y, Li G, Aluru NR. Physical models for coupled electromechanical analysis of silicon nanoelectromechanical systems. Journal of Applied Physics 2005; 97:114304(1-13).

[28] Chen H, Mukherjee S. Charge distribution on thin conducting nanotubes - reduced 3-D model. International Journal for Numerical Methods in Engineering, 68, 910-924, 2006.

[29] Harrington RF. Field Computation by Moment Methods. IEEE Press: Piscataway, NJ, 1993.

[30] Bao Z, Mukherjee S. Electrostatic BEM for MEMS with thin conducting plates and shells. Engineering Analysis with Boundary Elements 2004; 28:1427-1435.

[31] Bao Z, Mukherjee S. Electrostatic BEM for MEMS with thin beams. Communications in Numerical Methods in Engineering 2005; 21:297-312.

[32] Chuyan SW, Liao YS, Chen JT. Computational study of the effect of finger width and aspect ratios for the electrostatic levitating force of MEMS combdrive. Journal of Microelectromechanical Systems 2005; 14:305-312.

[33] Telukunta S, Mukherjee S. Fully Lagrangian modeling of MEMS with thin plates. Journal of Microelectromechanical Systems 2006; 15:795-810.

[34] Mukherjee S, Telukunta S, Mukherjee YX. BEM modeling of damping forces on MEMS with thin plates. Engineering Analysis with Boundary Elements 2005; 29:1000-1007. 
[35] Chen H, Mukherjee S. Modeling of the ground plane in electrostatic BEM analysis of MEMS and NEMS. Engineering Analysis with Boundary Elements 2006; 30:910-924.

[36] Mukherjee S. Finite parts of singular and hypersingular integrals with irregular boundary source points. Engineering Analysis with Boundary Element 2000; 24:767-776.

[37] Ma DDD, Lee CS, Au FCK, Tong SY, Lee ST. Small diameter silicon nanowire surfaces. Science 2003; 299:1874-1877.

[38] COMSOL, Inc. http://www.comsol.com/ 